\title{
Effects of Moxa (Artemisia vulgaris) Smoke Inhalation on Heart Rate and Its Variability
}

\author{
Baixiao Zhao ${ }^{1}$, Gerhard Litscher ${ }^{2}$, Jun Li $^{1}$, Lu Wang ${ }^{2}$, Yingxue Cui ${ }^{1}$, Chaxi Huang ${ }^{1}$, Ping Liu ${ }^{1}$ \\ ${ }^{1}$ School of Acupuncture-Moxibustion and Tuina, Beijing University of Chinese Medicine, Beijing, China \\ ${ }^{2}$ TCM Research Center Graz and Research Unit of Biomedical Engineering in Anesthesia \\ and Intensive Care Medicine, Medical University of Graz, Graz, Austria \\ E-mail: 1.14snowxu@163.com \\ Received March 24, 2011; revised April 1, 2011; accepted April 6, 2011
}

\begin{abstract}
Objective: To evaluate the changes of human heart rate (HR) and heart rate variability (HRV) during and after moxa smoke inhalation and to investigate the effects of moxa smoke on human autonomic nervous system. Methods: 24 healthy volunteers were exposed to moxa smoke with their HRV parameters measured before, during and after the moxa smoke inhalation. Results: The healthy volunteers exposed to moxa smoke had significant reductions in HR and also significant changes in HRV parameters. Conclusions: Moxa smoke can improve the autonomic nervous system activity. The inhalation of moxa smoke will induce a depressant effect on human body.
\end{abstract}

Keywords: Moxibustion, Moxa Smoke, Artemisia Vulgaris, Heart Rate (HR), Heart Rate Variability (HRV), Traditional Chinese Medicine

\section{Introduction}

Moxibustion, accompanied with herbal medicine and acupuncture, is one of the mainstream medical therapies in Traditional Chinese Medicine (TCM) with a history of more than 3000 years. Comparing with acupuncture, most of the common applications of moxibustion are non-invasive, thus it can be accepted by people more easily. As one of the major therapies in TCM, there exist a large amount of literature in the history of moxibustion which showed that moxibustion is applicable to many diseases, especially for chronic conditions, deficient pattern of a disease, prevention of disease, and health preservation. Modern researches have proven that moxibustion can improve the immune function of the body [1].

Since many people are exposed to moxa smoke during acupuncture-moxibustion treatment, some studies have been done recently to investigate if burning moxa in TCM constitutes a health hazard recently. There are no immediate concerns found arising from the continued use of moxa as therapeutic modality in TCM [2] However, to our knowledge the influence of moxa smoke on biological signals has not been investigated sufficiently in Asia and also in Europe [3,4].
Heart Rate Variability (HRV) refers to the time variation coefficient between successive heart beating cycles. It is a sensitive indicator of the autonomic nervous system and can reflect the activities of the sympathetic and parasympathetic nerves, the balance quality and the cardiovascular function. The objective of this study was to evaluate the changes of human heart rate (HR) and heart rate variability (HRV) after moxa smoke inhalation and to investigate the effects of moxa smoke on human autonomic nervous system.

\section{Materials and Methods}

\subsection{Healthy Volunteers}

The investigations were performed on 24 healthy volunteers (11 female, 13 male; aged 22 - 28 years old) from the Beijing University of Chinese Medicine. The subjects had no history of chronic diseases (such as hypertension, coronary heart disease or cerebrovascular disease), respiratory or neurological problems. On the day before the trial, the subjects were instructed to refrain from tobacco, alcohol, tea and coffee and to avoid strenuous exercise. They were fully informed about the nature of the investigation and all were given written informed consent. The 
methodological procedure and the registration of the non-invasive parameters were in accordance with the Helsinki Declaration of the World Medical Association.

\subsection{Physiological Measurements and Procedure}

The trial was carried out in two adjacent rooms equipped with massage beds at the Beijing University of Chinese Medicine. The environment around both rooms was quiet, and electrocardiographic parameters were registered in the subjects in a reclined position. The environmental conditions were kept constant (room temperature $20^{\circ} \mathrm{C}$, humidity $40 \%$ ) in both rooms. In addition, in room 2 , moxa floss (Artemisia vulgaris) was burnt producing moxa smoke. The concentration of the smoke was kept at a level comparable to that in acupuncture clinics (around $5 \mathrm{mg} / \mathrm{m}^{3}$ ) using a digital dusty indicator (Beijing BINTA Green. Technology co. Ltd, Beijing, China). The measurement procedure and the respective periods of rest and smoke inhalation can be seen in Figure 1.

An HRV medilog ${ }^{\circledR}$ AR12 (Huntleigh Healthcare, Cardiff, UK, and Leupamed GmbH, Graz, Austria) system was used for cardiac monitoring. The system is designed for long-term monitoring. The sampling rate of the new recorder is 4096 samples per second. Therefore RR-intervals can be detected extremely accurately. All raw data, stored digitally on special memory cards, were transferred via internet to the TCM Research Center Graz in Austria where the data analysis and the statistical analysis were performed.

HRV is measured as the percentage change in sequential chamber complexes, so-called RR-intervals, in the electrocardiogram (ECG). The registration of $\mathrm{HRV}$ is performed using three electrodes on the chest. Parameters used are those recommended by the Task Force of the European Society of Cardiology and the North American Society of Pacing and Electrophysiology [5]. Calculation of ECG power spectra is thought to provide an understanding of the effects of sympathetic and parasympathetic systems on HRV. Two main spectral components are distinguished in a spectrum calculated from short-term recordings of 5 minutes: low frequency (LF; $0.04-0.15 \mathrm{~Hz}$ ) and high frequency (HF; $0.15-0.4 \mathrm{~Hz}$ ) components. The LF/HF ratio has been accepted as an index of sympathovagal modulation of the sinoatrial node by the Task Force mentioned above [5]. In this pilot study, the evaluation parameters were: heart rate, total heart rate variability and the LF/HF ratio.

\subsection{Statistical Analysis}

The HR and HRV data were tested with one way repeated measures ANOVA. As post-hoc analysis, Tukey test was used (SigmaPlot 11.0, Systat Software Inc., Chicago, USA). The results were graphically presented as box plots. Changes were considered significant at a pvalue $<0.05$.

\section{Results}

All subjects completed the study. The electrocardiographic data of 24 subjects were transferred without any problems via internet over a distance of 7,650 km.

Figure 2 summarizes the moxa smoke induced changes of HR in all 24 volunteers. The results showed that significant $(\mathrm{p}<0.001)$ decreases in HR occur during moxa smoke inhalation. These effects remain present and intensified ( $\mathrm{p} \leq 0.039$ ) even ten minutes after completing the stimulation with moxa smoke.

The results of the total HRV of the volunteers $(n=24)$ are shown in Figure 3. A significant increase ( $\mathrm{p} \leq 0.037)$ of $\mathrm{HRV}_{\text {total }}$ after moxa smoke inhalation was observed as outstanding result of the beat-to-beat variability.

In addition to the global assessment of HRV, a separation into a LF and a HF-band was performed, and the LF/HF ratio was calculated. Figure 4 shows the results of this parameter. Insignificant changes were found from this analysis.

\section{Discussion}

The western understanding of "acupuncture” actually refers to the Chinese characters "Zhen Jiu”, which literally means acupuncture-moxibustion. This shows the enormous importance that moxibustion has in Chinese medicine since thousands of years. Moxibustion is composed

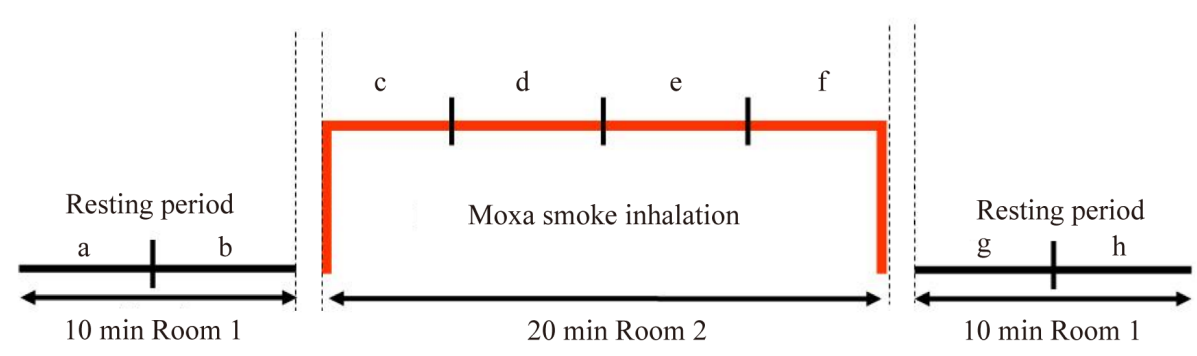

Figure 1. Measurement profile and measurement time periods ( $\mathrm{a}-\mathrm{h} ; \mathbf{5}$ minutes each). 


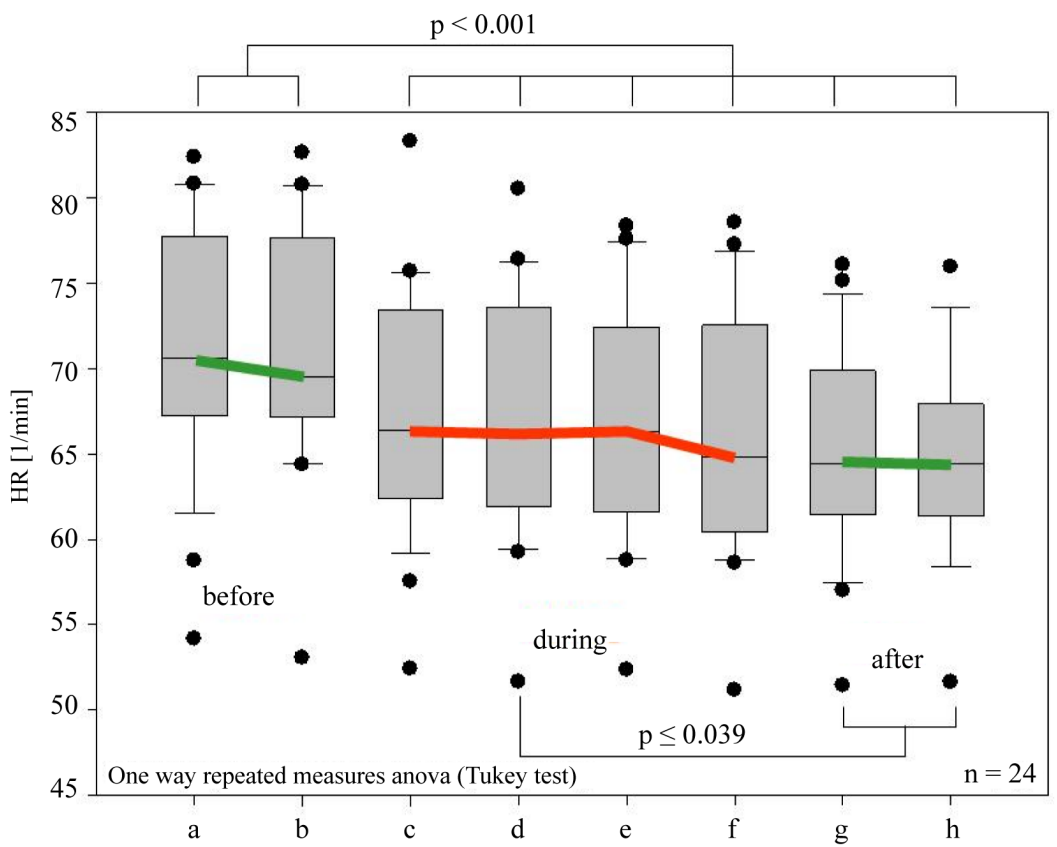

Figure 2. Box plot illustration of changes of heart rate (HR) in beats per minute in 24 healthy volunteers before (a,b), during (c-f) and after (g,h) moxa smoke inhalation. The horizontal line in the box gives the position of the median. The ends of the boxes define the $25^{\text {th }}$ and $75^{\text {th }}$ percentile; the error bars mark the $10^{\text {th }}$ and $90^{\text {th }}$ percentile.

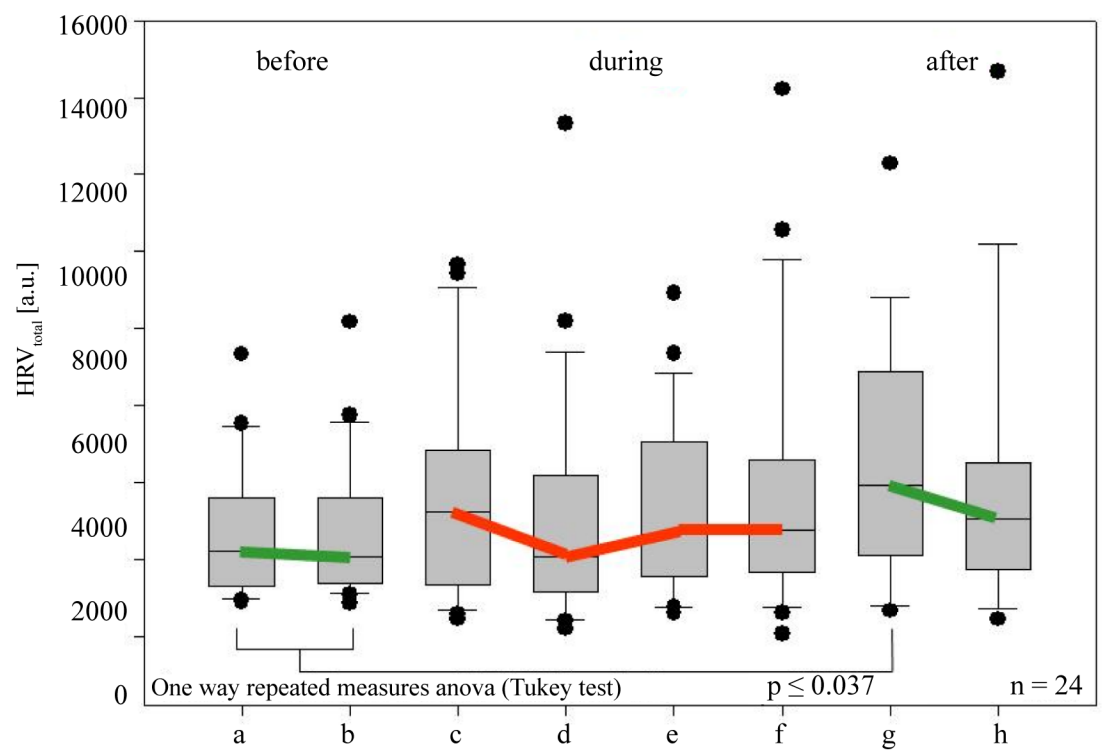

Figure 3. Changes of total heart rate variability $\left(\mathrm{HRV}_{\text {total }}\right)$ before, during and after moxa smoke inhalation. Note the significant increase in $\mathbf{H R V}_{\text {total }}$ after inhalation. Further explanations see Figure 2.

of the words mogusa (burning herb, mugwort is most commonly used) and "combustion” (burning). Mugwort is regarded as the best moxibustion material, established after thousands of years of practice. Comparing with the other materials for combustion, mugwort is inflammable and fragrant. The fire generated is moderate and gentle and suitable for treatment. When burning the mugwort, the heat given off can penetrate deeply into the muscles and bring about a comfortable feeling. There are also many other advantages of using mugwort such as extensive sources, simple mode of operation and safety.

It is reported in ancient books that the mugwort produced in Qizhou city, Hubei province is the best product. For the use of moxibustion, the mugwort leaves need to be harvested in a special season and placed in the shade to dry, then crush the leaves are crushed into moxa floss by re- 


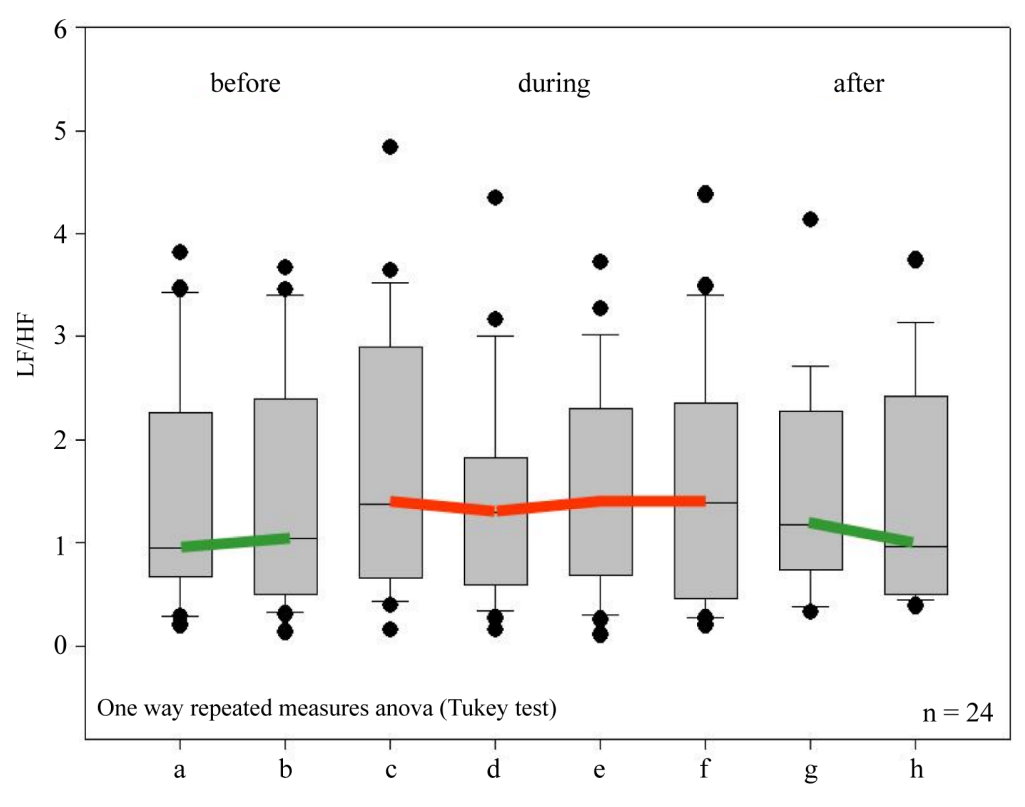

Figure 4. Ratio of the low (LF) and high (HF) frequency band of HRV in the 24 healthy volunteers before, during and after moxa smoke inhalation. No significant alterations were found. Further explanations see Figure 2.

moving the impurities. Good quality of mugwort floss should be soft and fine like cotton and can be stored for a long time. The longer the storage time, the better it is.

Mugwort leaf is also used as an herb for medication. Research showed that the main components of mugwort naphtha are eudesmol, thujone, camphor, borneo camphor, 4-terpene alcohol, caryophyllene, oleanolic acid, juniper camphor etc. [6]. These components may exist in the moxa smoke and have some treatment effects.

The RR-intervals in the ECG are controlled by the blood pressure control system, influenced by the hypothalamus and in particular controlled by the vagal cardiovascular center in the lower brainstem. HRV can be quantified over time using registration of percentage changes in RR-intervals in the time domain as well as the changes in the frequency range by analysis of electrocardiographic power spectra [7]. Earlier work done pointed out a few bands in the spectrum of HRV that could be interpreted as markers of physiological relevance. The total heart rate variability reflects the overall situation of HRV and the total autonomic nervous system activity .HF represents the parasympathetic nerve activity; LF represents the combined action of the parasympathetic nerve and the sympathetic nerve but mainly of the sympathetic nerve. Associated mechanisms are thermoregulation which can be found in the very low frequency band, blood pressure and respiratory effects $[5,7]$.

The present study revealed that the healthy volunteers exposed to moxa smoke had significant reductions in HR and also significant changes in HRV parameters, accompa- nied by a stress reduced tendency toward quiet behaviour. This shows that moxa smoke can improve the autonomic nervous system activity. The inhalation of moxa smoke will induce a depressant effect on human body.

This is in accordance with a preliminary study (diploma thesis) performed under supervision of the same institutions (Beijing University of Chinese Medicine and TCM Research Center Graz) [8]. In this research activity, a significant decrease of mean HR during treatment was observed in a total of 20 measurements using moxibustion on acupoint RN 4 (guān yuán) in two different sessions (needle-moxa and moxa-stick). The decreased HR then converged to baseline level again.

The possible reasons to account for the insignificant change in the LF/HF ratio in this study, which reflects the degree of balance between the two nervous systems, could be that the duration time of moxa smoke inhalation was not long enough and that the volunteers were already in a balanced health state. Further researches focused on patients and have longer observation time are needed. Beside these cardiovascular investigations, researches on other body systems, such as the neurophysiologic effects of moxa smoke inhalation, are absolutely necessary.

\section{Acknowledgements}

The authors thank Ms. Ingrid Gaischek, MSc, for her valuable support in creating figures and preparing the manuscript. The investigations were supported by the Austrian Ministries of Health and of Science and Research and the Eurasia-Pacific Uninet (project "Bioengi- 
neering and clinical assessment of high-tech acupuncture: a Sino-Austrian pilot study") and the Jubiläumsfonds of the Oesterreichische Nationalbank (OeNB; project 13463). The measurements and analysis were performed within the areas "Sustainable Health Research" and "Neuroscience" at the Medical University of Graz.

\section{References}

[1] C. L. Zhao, L. J. Peng, Z. L. Zhang, T. Zhang and H. M. $\mathrm{Li}$, "Effect of Acupuncture on the Activity of the Peripheral Blood T Lymphocyte Subsets and NK Cells in Patients with Colorectal Cancer Liver Metastasis,” Chinese Acupuncture \& Moxibustion, Vol. 30, No. 1, 2010, pp. 10-12.

[2] J. Wheeler, B. Coppock and C. Chen, "Does the Burning of Moxa (Artemisia Vulgaris) in Traditional Chinese Medicine Constitute a Health Hazard?” Acupuncture in Medicine, Vol. 27, No. 1, 2009, pp. 16-20. doi:10.1136/aim.2009.000422

[3] N. Hitosugi, R. Ohno, I. Hatsukari, S. Mizukami, H. Nagasaka, I. Matsumoto, N. Komatsu, M. Fujimaki, H. Nakashima, K. Satoh and H. Sakagami, "Diverse Biological
Activities of Moxa Extract and Smoke,” In Vivo, Vol. 15, No. 3, May-June 2001, pp. 249-254.

[4] N. Hitosugi, R. Ohno, I. Hatsukari, S. Nakamura, S. Mizukami, H. Nagasaka, I. Matsumoto, K. Satoh, T. Negoro, K. Hashimoto and H. Sakagami, "Induction of Cell Death by Pro-Oxidant Action of Moxa Smoke," Anticancer Research, Vol. 22, No. 1A, 2002, pp. 159-163.

[5] Task Force of the European Society of Cardiology and the North American Society of Pacing and Electrophysiology, "Heart Rate Variability: Standards of Measurement, Physiological Interpretation and Clinical Use,” European Heart Journal, Vol. 17, No. 3, 1996, pp. 354-381.

[6] R. Jin, M. M. Yu and B. X. Zhao, "Studies of Chemical Constituents of Qizhou Mugwort Leaf in Different Years and Mugwort Floss within Different Proportions," Chinese Acupuncture \& Moxibustion, Vol. 30, No. 5, 2010, pp. 389-392.

[7] G. Litscher, "Bioengineering Assessment of Acupuncture, Part 7: Heart Rate Variability," Critical Reviews in Biomedical Engineering, Vol. 35, No. 3, 2007, pp. 183-195.

[8] E. Hageneder, "Moxibustion-Aktuelle naturwissenschaftliche Betrachtungen,” Diploma Thesis, Medical University of Graz, Graz, 2010. 